\title{
Self adjoint operator harmonic polynomials induced Chebyshev-Grüss inequalities
}

George A. Anastassiou

\begin{abstract}
We present here very general self adjoint operator harmonic Chebyshev-Grüss inequalities with applications.

Mathematics Subject Classification (2010): 26D10, 26D20, 47A60, 47A67.

Keywords: Self adjoint operator, Hilbert space, Chebyshev-Grüss inequalities, harmonic polynomials.
\end{abstract}

\section{Motivation}

Here we mention the following inspiring and motivating result.

Theorem 1.1. (Čebyšev, 1882, [3]) Let $f, g:[a, b] \rightarrow \mathbb{R}$ absolutely continuous functions. If $f^{\prime}, g^{\prime} \in L_{\infty}([a, b])$, then

$$
\begin{aligned}
\mid \frac{1}{b-a} \int_{a}^{b} f(x) g(x) d x & -\left(\frac{1}{b-a} \int_{a}^{b} f(x) d x\right)\left(\frac{1}{b-a} \int_{a}^{b} g(x) d x\right) \mid \\
& \leq \frac{1}{12}(b-a)^{2}\left\|f^{\prime}\right\|_{\infty}\left\|g^{\prime}\right\|_{\infty} .
\end{aligned}
$$

Also we mention

Theorem 1.2. (Grüss, 1935, [9]) Let $f, g$ integrable functions from $[a, b]$ into $\mathbb{R}$, such that $m \leq f(x) \leq M, \rho \leq g(x) \leq \sigma$, for all $x \in[a, b]$, where $m, M, \rho, \sigma \in \mathbb{R}$. Then

$$
\begin{aligned}
\mid \frac{1}{b-a} \int_{a}^{b} f(x) g(x) d x & -\left(\frac{1}{b-a} \int_{a}^{b} f(x) d x\right)\left(\frac{1}{b-a} \int_{a}^{b} g(x) d x\right) \mid \\
& \leq \frac{1}{4}(M-m)(\sigma-\rho) .
\end{aligned}
$$

Next we follow [1], pp. 132-152.

We make 
Brief Assumption 1.3. Let $f: \prod_{i=1}^{m}\left[a_{i}, b_{i}\right] \rightarrow \mathbb{R}$ with $\frac{\partial^{l} f}{\partial x_{i}^{l}}$ for $l=0,1, \ldots, n ; i=1, \ldots, m$, are continuous on $\prod_{i=1}^{m}\left[a_{i}, b_{i}\right]$.

Definition 1.4. We put

$$
q\left(x_{i}, s_{i}\right)=\left\{\begin{array}{l}
s_{i}-a_{i}, \text { if } s_{i} \in\left[a_{i}, x_{i}\right] \\
s_{i}-b_{i}, \text { if } s_{i} \in\left(x_{i}, b_{i}\right]
\end{array}\right.
$$

$x_{i} \in\left[a_{i}, b_{i}\right], i=1, \ldots, m$.

Let $\left(P_{n}\right)_{n \in \mathbb{N}}$ be a harmonic sequence of polynomials, that is $P_{n}^{\prime}=P_{n-1}, n \in \mathbb{N}$, $P_{0}=1$.

Let functions $f_{\lambda}, \lambda=1, \ldots, r \in \mathbb{N}-\{1\}$, as in Brief Assumption 1.3, and $n_{\lambda} \in \mathbb{N}$ associated with $f_{\lambda}$.

We set

$$
\begin{gathered}
A_{i \lambda}\left(x_{i}, \ldots, x_{m}\right):=\frac{n_{\lambda}^{i-1}}{\prod_{j=1}^{i-1}\left(b_{j}-a_{j}\right)} \\
\times\left[\sum_{k=1}^{n_{\lambda}-1}(-1)^{k+1} P_{k}\left(x_{i}\right) \int_{a_{1}}^{b_{1}} \ldots \int_{a_{i-1}}^{b_{i-1}} \frac{\partial^{k} f_{\lambda}\left(s_{1}, \ldots, s_{i-1}, x_{i}, \ldots, x_{m}\right)}{\partial x_{i}^{k}} d s_{1} \ldots d s_{i-1}\right. \\
\quad+\sum_{k=1}^{n_{\lambda}-1} \frac{(-1)^{k}\left(n_{\lambda}-k\right)}{b_{i}-a_{i}} \\
\times\left[P_{k}\left(b_{i}\right) \int_{a_{1}}^{b_{1}} \ldots \int_{a_{i-1}}^{b_{i-1}} \frac{\partial^{k-1} f_{\lambda}\left(s_{1}, \ldots, s_{i-1}, b_{i}, x_{i+1}, \ldots, x_{m}\right)}{\partial x_{i}^{k-1}} d s_{1} \ldots d s_{i-1}\right. \\
\left.\left.-P_{k}\left(a_{i}\right) \int_{a_{1}}^{b_{1}} \ldots \int_{a_{i-1}}^{b_{i-1}} \frac{\partial^{k-1} f_{\lambda}\left(s_{1}, \ldots, s_{i-1}, a_{i}, x_{i+1}, \ldots, x_{m}\right)}{\partial x_{i}^{k-1}} d s_{1} \ldots d s_{i-1}\right]\right],
\end{gathered}
$$

and

$$
\begin{gathered}
B_{i \lambda}\left(x_{i}, \ldots, x_{m}\right):=\frac{n_{\lambda}^{i-1}(-1)^{n_{\lambda}+1}}{\prod_{j=1}^{i}\left(b_{j}-a_{j}\right)} \\
\times\left[\int_{a_{1}}^{b_{1}} \ldots \int_{a_{i}}^{b_{i}} P_{n_{\lambda}-1}\left(s_{i}\right) q\left(x_{i}, s_{i}\right) \frac{\partial^{n_{\lambda}} f_{\lambda}\left(s_{1}, \ldots, s_{i}, x_{i+1}, \ldots, x_{m}\right)}{\partial x_{i}^{n_{\lambda}}} d s_{1} \ldots d s_{i}\right],
\end{gathered}
$$

for all $i=1, \ldots, m ; \lambda=1, \ldots, r$.

We also set

$$
A_{1}:=\left(\frac{\left(\prod_{j=1}^{m}\left(b_{j}-a_{j}\right)\right)}{3}\right) \cdot\left[\sum _ { \lambda = 1 } ^ { r } \left\{\left(\prod_{\substack{\rho=1 \\ \rho \neq \lambda}}^{r}\left\|f_{\rho}\right\|_{\infty, \prod_{j=1}^{m}\left[a_{j}, b_{j}\right]}\right)\right.\right.
$$




$$
\left.\times\left(\sum_{i=1}^{m}\left[\left(b_{i}-a_{i}\right) n_{\lambda}^{i-1}\left\|P_{n_{\lambda}-1}\right\|_{\infty,\left[a_{i}, b_{i}\right]}\left\|\frac{\partial^{n_{\lambda}} f_{\lambda}}{\partial x_{i}^{n_{\lambda}}}\right\|_{\infty, \prod_{j=1}^{m}\left[a_{j}, b_{j}\right]}\right]\right)\right\},
$$

(let $p, q>1: \frac{1}{p}+\frac{1}{q}=1$ )

$$
A_{2}:=\sum_{\lambda=1}^{r} \sum_{i=1}^{m}\left\|\prod_{\substack{\rho=1 \\ \rho \neq \lambda}}^{r} f_{\rho}\right\|_{L_{p}\left(\prod_{j=1}^{m}\left[a_{j}, b_{j}\right]\right)}\left\|B_{i \lambda}\right\|_{L_{q}\left(\prod_{j=i}^{m}\left[a_{j}, b_{j}\right]\right)}\left(\prod_{j=1}^{i-1}\left(b_{j}-a_{j}\right)\right)^{\frac{1}{q}},
$$

and

$$
\begin{aligned}
A_{3}:= & \frac{1}{2}\left\{\sum _ { \lambda = 1 } ^ { r } \left\{\| \prod _ { \substack { \rho = 1 \\
\rho \neq \lambda } } ^ { r } f _ { \rho } \| _ { L _ { 1 } ( \prod _ { j = 1 } ^ { m } [ a _ { j } , b _ { j } ] ) } \left[\sum _ { i = 1 } ^ { m } \left[\left(b_{i}-a_{i}\right) n_{\lambda}^{i-1}\right.\right.\right.\right. \\
& \left.\left.\left.\left.\times\left\|P_{n_{\lambda}-1}\right\|_{\infty,\left[a_{i}, b_{i}\right]}\left\|\frac{\partial^{n_{\lambda}} f_{\lambda}}{\partial x_{i}^{n_{\lambda}}}\right\|_{\infty, \prod_{j=1}^{m}\left[a_{j}, b_{j}\right]}\right]\right]\right\}\right\} .
\end{aligned}
$$

We finally set

$$
\begin{gathered}
W:=r \int_{\prod_{j=1}^{m}\left[a_{j}, b_{j}\right]}\left(\prod_{\rho=1}^{r} f_{\rho}(x)\right) d x \\
-\frac{1}{\prod_{j=1}^{n}\left(b_{j}-a_{j}\right)} \sum_{\lambda=1}^{r} n_{\lambda}^{m}\left(\int_{\prod_{j=1}^{m}\left[a_{j}, b_{j}\right]}\left(\prod_{\substack{\rho=1 \\
\rho \neq \lambda}}^{r} f_{\rho}(x)\right) d x\right)\left(\int_{\prod_{j=1}^{m}\left[a_{j}, b_{j}\right]} f_{\lambda}(s) d s\right) \\
-\sum_{\lambda=1}^{r} \int_{\prod_{j=1}^{m}\left[a_{j}, b_{j}\right]}\left(\left(\prod_{\substack{\rho=1 \\
\rho \neq \lambda}}^{r} f_{\rho}(x)\right)\left(\sum_{i=1}^{m} A_{i \lambda}\left(x_{i}, \ldots, x_{m}\right)\right)\right) d x .
\end{gathered}
$$

We mention

Theorem 1.5. ([1], p. 151-152) It holds

$$
|W| \leq \min \left\{A_{1}, A_{2}, A_{3}\right\} .
$$

\section{Background}

Let $A$ be a selfadjoint linear operator on a complex Hilbert space $(H ;\langle\cdot, \cdot\rangle)$. The Gelfand map establishes a $*$-isometrically isomorphism $\Phi$ between the set $C(S p(A))$ 
of all continuous functions defined on the spectrum of $A$, denoted $S p(A)$, and the $C^{*}$ algebra $C^{*}(A)$ generated by $A$ and the identity operator $1_{H}$ on $H$ as follows (see e.g. $[8$, p. 3]):

For any $f, g \in C(S p(A))$ and any $\alpha, \beta \in \mathbb{C}$ we have

(i) $\Phi(\alpha f+\beta g)=\alpha \Phi(f)+\beta \Phi(g)$;

(ii) $\Phi(f g)=\Phi(f) \Phi(g)$ (the operation composition is on the right) and $\Phi(\bar{f})=(\Phi(f))^{*}$

(iii) $\|\Phi(f)\|=\|f\|:=\sup _{t \in S p(A)}|f(t)|$;

(iv) $\Phi\left(f_{0}\right)=1_{H}$ and $\Phi\left(f_{1}\right)=A$, where $f_{0}(t)=1$ and $f_{1}(t)=t$, for $t \in S p(A)$.

With this notation we define

$$
f(A):=\Phi(f), \text { for all } f \in C(S p(A)),
$$

and we call it the continuous functional calculus for a selfadjoint operator $A$.

If $A$ is a selfadjoint operator and $f$ is a real valued continuous function on $S p(A)$ then $f(t) \geq 0$ for any $t \in S p(A)$ implies that $f(A) \geq 0$, i.e. $f(A)$ is a positive operator on $H$. Moreover, if both $f$ and $g$ are real valued functions on $S p(A)$ then the following important property holds:

(P) $f(t) \geq g(t)$ for any $t \in S p(A)$, implies that $f(A) \geq g(A)$ in the operator order of $B(H)$.

Equivalently, we use (see [6], pp. 7-8):

Let $U$ be a selfadjoint operator on the complex Hilbert space $(H,\langle\cdot, \cdot\rangle)$ with the spectrum $S p(U)$ included in the interval $[m, M]$ for some real numbers $m<M$ and $\left\{E_{\lambda}\right\}_{\lambda}$ be its spectral family.

Then for any continuous function $f:[m, M] \rightarrow \mathbb{C}$, it is well known that we have the following spectral representation in terms of the Riemann-Stieljes integral:

$$
\langle f(U) x, y\rangle=\int_{m-0}^{M} f(\lambda) d\left(\left\langle E_{\lambda} x, y\right\rangle\right),
$$

for any $x, y \in H$. The function $g_{x, y}(\lambda):=\left\langle E_{\lambda} x, y\right\rangle$ is of bounded variation on the interval $[m, M]$, and

$$
g_{x, y}(m-0)=0 \text { and } g_{x, y}(M)=\langle x, y\rangle,
$$

for any $x, y \in H$. Furthermore, it is known that $g_{x}(\lambda):=\left\langle E_{\lambda} x, x\right\rangle$ is increasing and right continuous on $[m, M]$.

An important formula used a lot here is

$$
\langle f(U) x, x\rangle=\int_{m-0}^{M} f(\lambda) d\left(\left\langle E_{\lambda} x, x\right\rangle\right), \quad \forall x \in H .
$$

As a symbol we can write

$$
f(U)=\int_{m-0}^{M} f(\lambda) d E_{\lambda}
$$

Above,

$$
m=\min \{\lambda \mid \lambda \in S p(U)\}:=\min S p(U), M=\max \{\lambda \mid \lambda \in S p(U)\}:=\max S p(U) .
$$

The projections $\left\{E_{\lambda}\right\}_{\lambda \in \mathbb{R}}$, are called the spectral family of $A$, with the properties: 
(a) $E_{\lambda} \leq E_{\lambda^{\prime}}$ for $\lambda \leq \lambda^{\prime}$;

(b) $E_{m-0}=0_{H}$ (zero operator), $E_{M}=1_{H}$ (identity operator) and $E_{\lambda+0}=E_{\lambda}$ for all $\lambda \in \mathbb{R}$.

Furthermore

$$
E_{\lambda}:=\varphi_{\lambda}(U), \quad \forall \lambda \in \mathbb{R},
$$

is a projection which reduces $U$, with

$$
\varphi_{\lambda}(s):=\left\{\begin{array}{l}
1, \text { for }-\infty<s \leq \lambda, \\
0, \text { for } \lambda<s<+\infty .
\end{array}\right.
$$

The spectral family $\left\{E_{\lambda}\right\}_{\lambda \in \mathbb{R}}$ determines uniquely the self-adjoint operator $U$ and vice versa.

For more on the topic see [10], pp. 256-266, and for more detalis see there pp. 157-266. See also [5].

Some more basics are given (we follow [6], pp. 1-5):

Let $(H ;\langle\cdot, \cdot\rangle)$ be a Hilbert space over $\mathbb{C}$. A bounded linear operator $A$ defined on $H$ is selfjoint, i.e., $A=A^{*}$, iff $\langle A x, x\rangle \in \mathbb{R}, \forall x \in H$, and if $A$ is selfadjoint, then

$$
\|A\|=\sup _{x \in H:\|x\|=1}|\langle A x, x\rangle| .
$$

Let $A, B$ be selfadjoint operators on $H$. Then $A \leq B$ iff $\langle A x, x\rangle \leq\langle B x, x\rangle, \forall x \in H$.

In particular, $A$ is called positive if $A \geq 0$.

Denote by

$$
\mathcal{P}:=\left\{\varphi(s):=\sum_{k=0}^{n} \alpha_{k} s^{k} \mid n \geq 0, \alpha_{k} \in \mathbb{C}, 0 \leq k \leq n\right\} .
$$

If $A \in \mathcal{B}(H)$ (the Banach algebra of all bounded linear operators defined on $H$, i.e. from $H$ into itself) is selfadjoint, and $\varphi(s) \in \mathcal{P}$ has real coefficients, then $\varphi(A)$ is selfadjoint, and

$$
\|\varphi(A)\|=\max \{|\varphi(\lambda)|, \lambda \in S p(A)\} .
$$

If $\varphi$ is any function defined on $\mathbb{R}$ we define

$$
\|\varphi\|_{A}:=\sup \{|\varphi(\lambda)|, \lambda \in S p(A)\} .
$$

If $A$ is selfadjoint operator on Hilbert space $H$ and $\varphi$ is continuous and given that $\varphi(A)$ is selfadjoint, then $\|\varphi(A)\|=\|\varphi\|_{A}$. And if $\varphi$ is a continuous real valued function so it is $|\varphi|$, then $\varphi(A)$ and $|\varphi|(A)=|\varphi(A)|$ are selfadjoint operators (by [6], p. 4, Theorem 7).

Hence it holds

$$
\begin{aligned}
& \||\varphi(A)|\|=\||\varphi|\|_{A}=\sup \{\|\varphi(\lambda)\|, \lambda \in S p(A)\} \\
& =\sup \{|\varphi(\lambda)|, \lambda \in S p(A)\}=\|\varphi\|_{A}=\|\varphi(A)\|,
\end{aligned}
$$

that is

$$
\||\varphi(A)|\|=\|\varphi(A)\| .
$$

For a selfadjoint operator $A \in \mathcal{B}(H)$ which is positive, there exists a unique positive selfadjoint operator $B:=\sqrt{A} \in \mathcal{B}(H)$ such that $B^{2}=A$, that is $(\sqrt{A})^{2}=A$. We call $B$ the square root of $A$. 
Let $A \in \mathcal{B}(H)$, then $A^{*} A$ is selfadjoint and positive. Define the "operator absolute value" $|A|:=\sqrt{A^{*} A}$. If $A=A^{*}$, then $|A|=\sqrt{A^{2}}$.

For a continuous real valued function $\varphi$ we observe the following:

$$
\begin{gathered}
|\varphi(A)| \text { (the functional absolute value) }=\int_{m-0}^{M}|\varphi(\lambda)| d E_{\lambda} \\
=\int_{m-0}^{M} \sqrt{(\varphi(\lambda))^{2}} d E_{\lambda}=\sqrt{(\varphi(A))^{2}}=|\varphi(A)| \text { (operator absolute value), }
\end{gathered}
$$

where $A$ is a selfadjoint operator.

That is we have

$|\varphi(A)|$ (functional absolute value) $=|\varphi(A)|$ (operator absolute value).

Let $A, B \in \mathcal{B}(H)$, then

$$
\|A B\| \leq\|A\|\|B\|
$$

by Banach algebra property.

\section{Main results}

Let $\left(P_{n}\right)_{n \in \mathbb{N}}$ be a harmonic sequence of polynomials, that is $P_{n}^{\prime}=P_{n-1}, n \in \mathbb{N}$, $P_{0}=1$. Furthermore, let $[a, b] \subset \mathbb{R}, a \neq b$, and $h:[a, b] \rightarrow \mathbb{R}$ be such that $h^{(n-1)}$ is absolutely continuous function for some $n \in \mathbb{N}$.

We set

$$
q(x, t)=\left\{\begin{array}{ll}
t-a, & \text { if } t \in[a, x], \\
t-b, & \text { if } t \in(x, b],
\end{array} \quad x \in[a, b] .\right.
$$

By [4], and [1], p. 133, we get the generalized Fink type representation formula

$$
\begin{gathered}
h(x)=\sum_{k=1}^{n-1}(-1)^{k+1} P_{k}(x) h^{(k)}(x) \\
+\sum_{k=1}^{n-1} \frac{(-1)^{k}(n-k)}{b-a}\left[P_{k}(b) h^{(k-1)}(b)-P_{k}(a) h^{(k-1)}(a)\right] \\
+\frac{n}{b-a} \int_{a}^{b} h(t) d t+\frac{(-1)^{n+1}}{b-a} \int_{a}^{b} P_{n-1}(t) q(x, t) h^{(n)}(t) d t
\end{gathered}
$$

$\forall x \in[a, b], n \in \mathbb{N}$, when $n=1$ the above sums are zero.

For the harmonic sequence of polynomials $P_{k}(t)=\frac{(t-x)^{k}}{k !}, k \in \mathbb{Z}_{+},(3.2)$ reduces to Fink formula, see [7].

Next we present very general harmonic Chebyshev-Grüss operator inequalities based on (3.2). Then we specialize them for $n=1$.

We give 
Theorem 3.1. Let $n \in \mathbb{N}$ and $f, g \in C^{n}([a, b])$ with $[m, M] \subset(a, b), m<M$. Here $A$ is a selfadjoint linear bounded operator on the Hilbert space $H$ with spectrum $S p(A) \subseteq$ $[m, M]$. We consider any $x \in H:\|x\|=1$.

Then

$$
\begin{gathered}
\langle(\Delta(f, g))(A) x, x\rangle:=\mid\langle f(A) g(A) x, x\rangle-\langle f(A) x, x\rangle\langle g(A) x, x\rangle \\
-\frac{1}{2}\left[\sum _ { k = 1 } ^ { n - 1 } ( - 1 ) ^ { k + 1 } \left\{\left[\left\langle P_{k}(A)\left(g(A) f^{(k)}(A)+f(A) g^{(k)}(A)\right) x, x\right\rangle\right]\right.\right. \\
\left.\left.-\left[\left\langle P_{k}(A) f^{(k)}(A) x, x\right\rangle\langle g(A) x, x\rangle+\left\langle P_{k}(A) g^{(k)}(A) x, x\right\rangle\langle f(A) x, x\rangle\right]\right\}\right] \mid \\
\leq \frac{\left[\|g(A)\|\left\|f^{(n)}\right\|_{\infty,[m, M]}+\|f(A)\|\left\|g^{(n)}\right\|_{\infty,[m, M]}\right]}{2(M-m)} \\
\left\|P_{n-1}\right\|_{\infty,[m, M]}\left[\left\|\left(M 1_{H}-A\right)^{2}\right\|+\left\|\left(A-m 1_{H}\right)^{2}\right\|\right] .
\end{gathered}
$$

Proof. Here $\left\{E_{\lambda}\right\}_{\lambda \in \mathbb{R}}$ is the spectral family of $A$. Set

$$
k(\lambda, t):= \begin{cases}t-m, & m \leq t \leq \lambda, \\ t-M, & \lambda<t \leq M .\end{cases}
$$

where $\lambda \in[m, M]$.

Hence by (3.2) we obtain

$$
\begin{gathered}
f(\lambda)=\sum_{k=1}^{n-1}(-1)^{k+1} P_{k}(\lambda) f^{(k)}(\lambda) \\
+\sum_{k=1}^{n-1} \frac{(-1)^{k}(n-k)}{M-m}\left[P_{k}(M) f^{(k-1)}(M)-P_{k}(m) f^{(k-1)}(m)\right] \\
+\frac{n}{M-m} \int_{m}^{M} f(t) d t+\frac{(-1)^{n+1}}{M-m} \int_{m}^{M} P_{n-1}(t) k(\lambda, t) f^{(n)}(t) d t,
\end{gathered}
$$

and

$$
\begin{gathered}
g(\lambda)=\sum_{k=1}^{n-1}(-1)^{k+1} P_{k}(\lambda) g^{(k)}(\lambda) \\
+\sum_{k=1}^{n-1} \frac{(-1)^{k}(n-k)}{M-m}\left[P_{k}(M) g^{(k-1)}(M)-P_{k}(m) g^{(k-1)}(m)\right] \\
+\frac{n}{M-m} \int_{m}^{M} g(t) d t+\frac{(-1)^{n+1}}{M-m} \int_{m}^{M} P_{n-1}(t) k(\lambda, t) g^{(n)}(t) d t
\end{gathered}
$$

$\forall \lambda \in[m, M]$.

By applying the spectral representation theorem on (3.5), (3.6), i.e. integrating against $E_{\lambda}$ over $[m, M]$, see $(2.3)$, (ii), we obtain:

$$
f(A)=\sum_{k=1}^{n-1}(-1)^{k+1} P_{k}(A) f^{(k)}(A)
$$




$$
\begin{gathered}
+\left(\sum_{k=1}^{n-1} \frac{(-1)^{k}(n-k)}{M-m}\left[P_{k}(M) f^{(k-1)}(M)-P_{k}(m) f^{(k-1)}(m)\right]\right) 1_{H} \\
+\left(\frac{n}{M-m} \int_{m}^{M} f(t) d t\right) 1_{H}+\frac{(-1)^{n+1}}{M-m} \int_{m-0}^{M}\left(\int_{m}^{M} P_{n-1}(t) k(\lambda, t) f^{(n)}(t) d t\right) d E_{\lambda},
\end{gathered}
$$

and

$$
\begin{gathered}
g(A)=\sum_{k=1}^{n-1}(-1)^{k+1} P_{k}(A) g^{(k)}(A) \\
+\left(\sum_{k=1}^{n-1} \frac{(-1)^{k}(n-k)}{M-m}\left[P_{k}(M) g^{(k-1)}(M)-P_{k}(m) g^{(k-1)}(m)\right]\right) 1_{H} \\
+\left(\frac{n}{M-m} \int_{m}^{M} g(t) d t\right) 1_{H}+\frac{(-1)^{n+1}}{M-m} \int_{m-0}^{M}\left(\int_{m}^{M} P_{n-1}(t) k(\lambda, t) g^{(n)}(t) d t\right) d E_{\lambda} .
\end{gathered}
$$

We notice that

$$
g(A) f(A)=f(A) g(A)
$$

to be used next.

Then it holds

$$
\begin{gathered}
g(A) f(A)=\sum_{k=1}^{n-1}(-1)^{k+1} g(A) P_{k}(A) f^{(k)}(A) \\
+\left(\sum_{k=1}^{n-1} \frac{(-1)^{k}(n-k)}{M-m}\left[P_{k}(M) f^{(k-1)}(M)-P_{k}(m) f^{(k-1)}(m)\right]\right) g(A) \\
+\left(\frac{n}{M-m} \int_{m}^{M} f(t) d t\right) g(A) \\
+\frac{(-1)^{n+1}}{M-m} g(A) \int_{m-0}^{M}\left(\int_{m}^{M} P_{n-1}(t) k(\lambda, t) f^{(n)}(t) d t\right) d E_{\lambda},
\end{gathered}
$$

and

$$
\begin{gathered}
f(A) g(A)=\sum_{k=1}^{n-1}(-1)^{k+1} f(A) P_{k}(A) g^{(k)}(A) \\
+\left(\sum_{k=1}^{n-1} \frac{(-1)^{k}(n-k)}{M-m}\left[P_{k}(M) g^{(k-1)}(M)-P_{k}(m) g^{(k-1)}(m)\right]\right) f(A) \\
+\left(\frac{n}{M-m} \int_{m}^{M} g(t) d t\right) f(A) \\
+\frac{(-1)^{n+1}}{M-m} f(A) \int_{m-0}^{M}\left(\int_{m}^{M} P_{n-1}(t) k(\lambda, t) g^{(n)}(t) d t\right) d E_{\lambda} .
\end{gathered}
$$


Here from now on we consider $x \in H:\|x\|=1$; immediately we get

$$
\int_{m-0}^{M} d\left\langle E_{\lambda} x, x\right\rangle=1 .
$$

Then it holds (see (2.2))

$$
\begin{gathered}
\langle f(A) x, x\rangle=\sum_{k=1}^{n-1}(-1)^{k+1}\left\langle P_{k}(A) f^{(k)}(A) x, x\right\rangle \\
+\sum_{k=1}^{n-1} \frac{(-1)^{k}(n-k)}{M-m}\left[P_{k}(M) f^{(k-1)}(M)-P_{k}(m) f^{(k-1)}(m)\right] \\
+\frac{n}{M-m} \int_{m}^{M} f(t) d t+\frac{(-1)^{n+1}}{M-m} \int_{m-0}^{M}\left(\int_{m}^{M} P_{n-1}(t) k(\lambda, t) f^{(n)}(t) d t\right) d\left\langle E_{\lambda} x, x\right\rangle,
\end{gathered}
$$

and

$$
\begin{gathered}
\langle g(A) x, x\rangle=\sum_{k=1}^{n-1}(-1)^{k+1}\left\langle P_{k}(A) g^{(k)}(A) x, x\right\rangle \\
+\sum_{k=1}^{n-1} \frac{(-1)^{k}(n-k)}{M-m}\left[P_{k}(M) g^{(k-1)}(M)-P_{k}(m) g^{(k-1)}(m)\right] \\
+\frac{n}{M-m} \int_{m}^{M} g(t) d t+\frac{(-1)^{n+1}}{M-m} \int_{m-0}^{M}\left(\int_{m}^{M} P_{n-1}(t) k(\lambda, t) g^{(n)}(t) d t\right) d\left\langle E_{\lambda} x, x\right\rangle .
\end{gathered}
$$

Then we get

$$
\begin{gathered}
\langle f(A) x, x\rangle\langle g(A) x, x\rangle=\sum_{k=1}^{n-1}(-1)^{k+1}\left\langle P_{k}(A) f^{(k)}(A) x, x\right\rangle\langle g(A) x, x\rangle \\
+\left(\sum_{k=1}^{n-1} \frac{(-1)^{k}(n-k)}{M-m}\left[P_{k}(M) f^{(k-1)}(M)-P_{k}(m) f^{(k-1)}(m)\right]\right)\langle g(A) x, x\rangle \\
+\left(\frac{n}{M-m} \int_{m}^{M} f(t) d t\right)\langle g(A) x, x\rangle \\
+\frac{(-1)^{n+1}\langle g(A) x, x\rangle}{M-m} \int_{m-0}^{M}\left(\int_{m}^{M} P_{n-1}(t) k(\lambda, t) f^{(n)}(t) d t\right) d\left\langle E_{\lambda} x, x\right\rangle
\end{gathered}
$$

and

$$
\begin{gathered}
\langle g(A) x, x\rangle\langle f(A) x, x\rangle=\sum_{k=1}^{n-1}(-1)^{k+1}\left\langle P_{k}(A) g^{(k)}(A) x, x\right\rangle\langle f(A) x, x\rangle \\
+\left(\sum_{k=1}^{n-1} \frac{(-1)^{k}(n-k)}{M-m}\left[P_{k}(M) g^{(k-1)}(M)-P_{k}(m) g^{(k-1)}(m)\right]\right)\langle f(A) x, x\rangle \\
+\left(\frac{n}{M-m} \int_{m}^{M} g(t) d t\right)\langle f(A) x, x\rangle
\end{gathered}
$$




$$
+\frac{(-1)^{n+1}\langle f(A) x, x\rangle}{M-m} \int_{m-0}^{M}\left(\int_{m}^{M} P_{n-1}(t) k(\lambda, t) g^{(n)}(t) d t\right) d\left\langle E_{\lambda} x, x\right\rangle .
$$

Furthermore we obtain

$$
\begin{gathered}
\langle f(A) g(A) x, x\rangle \stackrel{(3.10)}{=} \sum_{k=1}^{n-1}(-1)^{k+1}\left\langle g(A) P_{k}(A) f^{(k)}(A) x, x\right\rangle \\
+\left(\sum_{k=1}^{n-1} \frac{(-1)^{k}(n-k)}{M-m}\left[P_{k}(M) f^{(k-1)}(M)-P_{k}(m) f^{(k-1)}(m)\right]\right)\langle g(A) x, x\rangle \\
+\left(\frac{n}{M-m} \int_{m}^{M} f(t) d t\right)\langle g(A) x, x\rangle \\
+\frac{(-1)^{n+1}}{M-m}\left\langle\left(g(A) \int_{m-0}^{M}\left(\int_{m}^{M} P_{n-1}(t) k(\lambda, t) f^{(n)}(t) d t\right) d E_{\lambda}\right) x, x\right\rangle
\end{gathered}
$$

and

$$
\begin{gathered}
\langle f(A) g(A) x, x\rangle \stackrel{(3.11)}{=} \sum_{k=1}^{n-1}(-1)^{k+1}\left\langle f(A) P_{k}(A) g^{(k)}(A) x, x\right\rangle \\
+\left(\sum_{k=1}^{n-1} \frac{(-1)^{k}(n-k)}{M-m}\left[P_{k}(M) g^{(k-1)}(M)-P_{k}(m) g^{(k-1)}(m)\right]\right)\langle f(A) x, x\rangle \\
+\left(\frac{n}{M-m} \int_{m}^{M} g(t) d t\right)\langle f(A) x, x\rangle \\
+\frac{(-1)^{n+1}}{M-m}\left\langle\left(f(A) \int_{m-0}^{M}\left(\int_{m}^{M} P_{n-1}(t) k(\lambda, t) g^{(n)}(t) d t\right) d E_{\lambda}\right) x, x\right\rangle
\end{gathered}
$$

By (3.14) and (3.16) we obtain

$$
\begin{gathered}
E:=\langle f(A) g(A) x, x\rangle-\langle f(A) x, x\rangle\langle g(A) x, x\rangle \\
=\sum_{k=1}^{n-1}(-1)^{k+1}\left[\left\langle g(A) P_{k}(A) f^{(k)}(A) x, x\right\rangle-\left\langle P_{k}(A) f^{(k)}(A) x, x\right\rangle\langle g(A) x, x\rangle\right] \\
+\frac{(-1)^{n+1}}{M-m}\left[\left\langle\left(g(A) \int_{m-0}^{M}\left(\int_{m}^{M} P_{n-1}(t) k(\lambda, t) f^{(n)}(t) d t\right) d E_{\lambda}\right) x, x\right\rangle\right. \\
\left.-\langle g(A) x, x\rangle \int_{m-0}^{M}\left(\int_{m}^{M} P_{n-1}(t) k(\lambda, t) f^{(n)}(t) d t\right) d\left\langle E_{\lambda} x, x\right\rangle\right],
\end{gathered}
$$

and by (3.15) and (3.17) we derive

$$
\begin{gathered}
E:=\langle f(A) g(A) x, x\rangle-\langle f(A) x, x\rangle\langle g(A) x, x\rangle \\
=\sum_{k=1}^{n-1}(-1)^{k+1}\left[\left\langle f(A) P_{k}(A) g^{(k)}(A) x, x\right\rangle-\left\langle P_{k}(A) g^{(k)}(A) x, x\right\rangle\langle f(A) x, x\rangle\right]
\end{gathered}
$$




$$
\begin{gathered}
+\frac{(-1)^{n+1}}{M-m}\left[\left\langle\left(f(A) \int_{m-0}^{M}\left(\int_{m}^{M} P_{n-1}(t) k(\lambda, t) g^{(n)}(t) d t\right) d E_{\lambda}\right) x, x\right\rangle\right. \\
\left.=\langle f(A) x, x\rangle \int_{m-0}^{M}\left(\int_{m}^{M} P_{n-1}(t) k(\lambda, t) g^{(n)}(t) d t\right) d\left\langle E_{\lambda} x, x\right\rangle\right] .
\end{gathered}
$$

Consequently, we get that

$$
\begin{aligned}
2 E & =\sum_{k=1}^{n-1}(-1)^{k+1}\left\{\left[\left\langle g(A) P_{k}(A) f^{(k)}(A) x, x\right\rangle+\left\langle f(A) P_{k}(A) g^{(k)}(A) x, x\right\rangle\right]\right. \\
- & {\left.\left[\left\langle P_{k}(A) f^{(k)}(A) x, x\right\rangle\langle g(A) x, x\rangle+\left\langle P_{k}(A) g^{(k)}(A) x, x\right\rangle\langle f(A) x, x\rangle\right]\right\} } \\
+ & \frac{(-1)^{n+1}}{M-m}\left\{\left[\left\langle\left(g(A) \int_{m-0}^{M}\left(\int_{m}^{M} P_{n-1}(t) k(\lambda, t) f^{(n)}(t) d t\right) d E_{\lambda}\right) x, x\right\rangle\right.\right. \\
+ & \left.\left\langle\left(f(A) \int_{m-0}^{M}\left(\int_{m}^{M} P_{n-1}(t) k(\lambda, t) g^{(n)}(t) d t\right) d E_{\lambda}\right) x, x\right\rangle\right] \\
& -\left[\langle g(A) x, x\rangle \int_{m-0}^{M}\left(\int_{m}^{M} P_{n-1}(t) k(\lambda, t) f^{(n)}(t) d t\right) d\left\langle E_{\lambda} x, x\right\rangle\right. \\
+ & \left.\left.\langle f(A) x, x\rangle \int_{m-0}^{M}\left(\int_{m}^{M} P_{n-1}(t) k(\lambda, t) g^{(n)}(t) d t\right) d\left\langle E_{\lambda} x, x\right\rangle\right]\right\} .
\end{aligned}
$$

We find that

$$
\begin{gathered}
\langle f(A) g(A) x, x\rangle-\langle f(A) x, x\rangle\langle g(A) x, x\rangle \\
-\frac{1}{2}\left[\sum _ { k = 1 } ^ { n - 1 } ( - 1 ) ^ { k + 1 } \left\{\left[\left\langle P_{k}(A)\left(g(A) f^{(k)}(A)+f(A) g^{(k)}(A)\right) x, x\right\rangle\right]\right.\right. \\
\left.\left.-\left[\left\langle P_{k}(A) f^{(k)}(A) x, x\right\rangle\langle g(A) x, x\rangle+\left\langle P_{k}(A) g^{(k)}(A) x, x\right\rangle\langle f(A) x, x\rangle\right]\right\}\right] \\
=\frac{(-1)^{n+1}}{2(M-m)}\left\{\left[\left\langle\left(g(A) \int_{m-0}^{M}\left(\int_{m}^{M} P_{n-1}(t) k(\lambda, t) f^{(n)}(t) d t\right) d E_{\lambda}\right) x, x\right\rangle\right.\right. \\
\left.+\left\langle\left(f(A) \int_{m-0}^{M}\left(\int_{m}^{M} P_{n-1}(t) k(\lambda, t) g^{(n)}(t) d t\right) d E_{\lambda}\right) x, x\right\rangle\right] \\
-\left[\langle g(A) x, x\rangle \int_{m-0}^{M}\left(\int_{m}^{M} P_{n-1}(t) k(\lambda, t) f^{(n)}(t) d t\right) d\left\langle E_{\lambda} x, x\right\rangle\right. \\
\left.\left.+\langle f(A) x, x\rangle \int_{m-0}^{M}\left(\int_{m}^{M} P_{n-1}(t) k(\lambda, t) g^{(n)}(t) d t\right) d\left\langle E_{\lambda} x, x\right\rangle\right]\right\}=: R .
\end{gathered}
$$

Therefore it holds

$$
|R| \leq \frac{1}{2(M-m)}\left\{\left[\|g(A)\|\left\|\int_{m-0}^{M}\left(\int_{m}^{M} P_{n-1}(t) k(\lambda, t) f^{(n)}(t) d t\right) d E_{\lambda}\right\|\right.\right.
$$




$$
\begin{gathered}
\left.+\|f(A)\|\left\|\int_{m-0}^{M}\left(\int_{m}^{M} P_{n-1}(t) k(\lambda, t) g^{(n)}(t) d t\right) d E_{\lambda}\right\|\right] \\
+\left[\|g(A)\|\left\|\int_{m-0}^{M}\left(\int_{m}^{M} P_{n-1}(t) k(\lambda, t) f^{(n)}(t) d t\right) d E_{\lambda}\right\|\right. \\
\left.\left.+\|f(A)\|\left\|\int_{m-0}^{M}\left(\int_{m}^{M} P_{n-1}(t) k(\lambda, t) g^{(n)}(t) d t\right) d E_{\lambda}\right\|\right]\right\} \\
=\frac{1}{(M-m)}\left\{\|g(A)\|\left\|\int_{m-0}^{M}\left(\int_{m}^{M} P_{n-1}(t) k(\lambda, t) f^{(n)}(t) d t\right) d E_{\lambda}\right\|\right. \\
\left.+\|f(A)\|\left\|\int_{m-0}^{M}\left(\int_{m}^{M} P_{n-1}(t) k(\lambda, t) g^{(n)}(t) d t\right) d E_{\lambda}\right\|\right\}=:\left(\xi_{1}\right) .
\end{gathered}
$$

We notice the following:

$$
\begin{aligned}
&\left\|\int_{m-0}^{M}\left(\int_{m}^{M} P_{n-1}(t) k(\lambda, t) f^{(n)}(t) d t\right) d E_{\lambda}\right\| \\
&=\sup _{x \in H:\|x\|=1} \mid \int_{m-0}^{M}\left(\int_{m}^{M} P_{n-1}(t) k(\lambda, t) f^{(n)}(t) d t\right) d\left\langle E_{\lambda} x, x\right\rangle \mid \\
& \leq \sup _{x \in H:\|x\|=1}\left(\int_{m-0}^{M}\right.\left.\left(\int_{m}^{M}\left|P_{n-1}(t)\right||k(\lambda, t)|\left|f^{(n)}(t)\right| d t\right) d\left\langle E_{\lambda} x, x\right\rangle\right) \\
& \leq\left(\left\|P_{n-1}\right\|_{\infty,[m, M]}\left\|f^{(n)}\right\|_{\infty,[m, M]}\right) \\
& \sup _{x \in H:\|x\|=1}\left(\int_{m-0}^{M}\left(\int_{m}^{M}|k(\lambda, t)| d t\right) d\left\langle E_{\lambda} x, x\right\rangle\right)=:\left(\xi_{2}\right) .
\end{aligned}
$$

(Notice that

$$
\left.\int_{m}^{M}|k(\lambda, t)| d t=\int_{m}^{\lambda}(t-m) d t+\int_{\lambda}^{M}(M-t) d t=\frac{(\lambda-m)^{2}+(M-\lambda)^{2}}{2} .\right)
$$

Hence it holds

$$
\begin{gathered}
\left(\xi_{2}\right) \stackrel{(3.25)}{=}\left(\frac{\left\|P_{n-1}\right\|_{\infty,[m, M]}\left\|f^{(n)}\right\|_{\infty,[m, M]}}{2}\right) \\
\times \sup _{x \in H:\|x\|=1}\left[\left\langle\left(M 1_{H}-A\right)^{2} x, x\right\rangle+\left\langle\left(A-m 1_{H}\right)^{2} x, x\right\rangle\right] \\
\leq\left(\frac{\left\|P_{n-1}\right\|_{\infty,[m, M]}\left\|f^{(n)}\right\|_{\infty,[m, M]}}{2}\right)\left[\left\|\left(M 1_{H}-A\right)^{2}\right\|+\left\|\left(A-m 1_{H}\right)^{2}\right\|\right] .
\end{gathered}
$$

We have proved that

$$
\left\|\int_{m-0}^{M}\left(\int_{m}^{M} P_{n-1}(t) k(\lambda, t) f^{(n)}(t) d t\right) d E_{\lambda}\right\|
$$




$$
\leq\left(\frac{\left\|P_{n-1}\right\|_{\infty,[m, M]}\left\|f^{(n)}\right\|_{\infty,[m, M]}}{2}\right)\left[\left\|\left(M 1_{H}-A\right)^{2}\right\|+\left\|\left(A-m 1_{H}\right)^{2}\right\|\right] .
$$

Similarly, it holds

$$
\begin{gathered}
\left\|\int_{m-0}^{M}\left(\int_{m}^{M} P_{n-1}(t) k(\lambda, t) g^{(n)}(t) d t\right) d E_{\lambda}\right\| \\
\leq\left(\frac{\left\|P_{n-1}\right\|_{\infty,[m, M]}\left\|g^{(n)}\right\|_{\infty,[m, M]}}{2}\right)\left[\left\|\left(M 1_{H}-A\right)^{2}\right\|+\left\|\left(A-m 1_{H}\right)^{2}\right\|\right] .
\end{gathered}
$$

Next we apply (3.27), (3.28) into (3.23), we get

$$
\begin{gathered}
\left(\xi_{1}\right) \leq \frac{1}{(M-m)}\left\{\| g ( A ) \| \left(\frac{\left\|P_{n-1}\right\|_{\infty,[m, M]}\left\|f^{(n)}\right\|_{\infty,[m, M]}}{2}\right.\right. \\
\left.\times\left[\left\|\left(M 1_{H}-A\right)^{2}\right\|+\left\|\left(A-m 1_{H}\right)^{2}\right\|\right]\right)+\|f(A)\| \\
\left.\times\left(\frac{\left\|P_{n-1}\right\|_{\infty,[m, M]}\left\|g^{(n)}\right\|_{\infty,[m, M]}}{2}\right)\left[\left\|\left(M 1_{H}-A\right)^{2}\right\|+\left\|\left(A-m 1_{H}\right)^{2}\right\|\right]\right\} \\
=\frac{1}{2(M-m)}\left\{\left[\|g(A)\|\left\|f^{(n)}\right\|_{\infty,[m, M]}+\|f(A)\|\left\|g^{(n)}\right\|_{\infty,[m, M]}\right]\right. \\
\left.\left\|P_{n-1}\right\|_{\infty,[m, M]}\left[\left\|\left(M 1_{H}-A\right)^{2}\right\|+\left\|\left(A-m 1_{H}\right)^{2}\right\|\right]\right\} .
\end{gathered}
$$

We have proved that

$$
\begin{gathered}
|R| \leq \frac{\left(\|g(A)\|\left\|f^{(n)}\right\|_{\infty,[m, M]}+\|f(A)\|\left\|g^{(n)}\right\|_{\infty,[m, M]}\right)}{2(M-m)} \\
\left\|P_{n-1}\right\|_{\infty,[m, M]}\left[\left\|\left(M 1_{H}-A\right)^{2}\right\|+\left\|\left(A-m 1_{H}\right)^{2}\right\|\right] .
\end{gathered}
$$

The theorem is proved.

It follows the case $n=1$.

Corollary 3.2. (to Theorem 3.1) Let $f, g \in C^{1}([a, b])$ with $[m, M] \subset(a, b), m<M$. Here $A$ is a selfadjoint bounded linear operator on the Hilbert space $H$ with spectrum $S p(A) \subseteq[m, M]$. We consider any $x \in H:\|x\|=1$.

Then

$$
\begin{gathered}
\leq|\langle f(A) g(A) x, x\rangle-\langle f(A) x, x\rangle\langle g(A) x, x\rangle| \\
\leq \frac{\left[\|g(A)\|\left\|f^{\prime}\right\|_{\infty,[m, M]}+\|f(A)\|\left\|g^{\prime}\right\|_{\infty,[m, M]}\right]}{2(M-m)} \\
{\left[\left\|\left(M 1_{H}-A\right)^{2}\right\|+\left\|\left(A-m 1_{H}\right)^{2}\right\|\right] .}
\end{gathered}
$$

We continue with 
Theorem 3.3. All as in Theorem 3.1. Let $\alpha, \beta, \gamma>1: \frac{1}{\alpha}+\frac{1}{\beta}+\frac{1}{\gamma}=1$. Then

$$
\begin{gathered}
\langle(\Delta(f, g))(A) x, x\rangle \leq \frac{\left\|P_{n-1}\right\|_{\alpha,[m, M]}}{(M-m)(\beta+1)^{\frac{1}{\beta}}} \\
{\left[\|g(A)\|\left\|f^{(n)}\right\|_{\gamma,[m, M]}+\|f(A)\|\left\|g^{(n)}\right\|_{\gamma,[m, M]}\right]} \\
{\left[\left\|\left(A-m 1_{H}\right)^{1+\frac{1}{\beta}}\right\|+\left\|\left(M 1_{H}-A\right)^{1+\frac{1}{\beta}}\right\|\right] .}
\end{gathered}
$$

Proof. As in (3.24) we have

$$
\begin{gathered}
\\
\qquad \int_{m-0}^{M}\left(\int_{m}^{M} P_{n-1}(t) k(\lambda, t) f^{(n)}(t) d t\right) d E_{\lambda} \| \\
=\sup _{x \in H:\|x\|=1}\left|\int_{m-0}^{M}\left(\int_{m}^{M} P_{n-1}(t) k(\lambda, t) f^{(n)}(t) d t\right) d\left(\left\langle E_{\lambda} x, x\right\rangle\right)\right|=: \psi_{1} .
\end{gathered}
$$

Here $\alpha, \beta, \gamma>1: \frac{1}{\alpha}+\frac{1}{\beta}+\frac{1}{\gamma}=1$. By Hölder's inequality for three functions we get

$$
\begin{gathered}
\left|\int_{m}^{M} P_{n-1}(t) k(\lambda, t) f^{(n)}(t) d t\right| \leq \int_{m}^{M}\left|P_{n-1}(t)\right||k(\lambda, t)|\left|f^{(n)}(t)\right| d t \\
\leq\left\|P_{n-1}\right\|_{\alpha}\left\|f^{(n)}\right\|_{\gamma}\left(\int_{m}^{M}|k(\lambda, t)|^{\beta} d t\right)^{\frac{1}{\beta}} \\
=\left\|P_{n-1}\right\|_{\alpha}\left\|f^{(n)}\right\|_{\gamma}\left(\int_{m}^{\lambda}(t-m)^{\beta} d t+\int_{\lambda}^{M}(M-t)^{\beta} d t\right)^{\frac{1}{\beta}} \\
=\left\|P_{n-1}\right\|_{\alpha}\left\|f^{(n)}\right\|_{\gamma}\left[\frac{(\lambda-m)^{\beta+1}+(M-\lambda)^{\beta+1}}{\beta+1}\right]^{\frac{1}{\beta}} \\
\leq \frac{\left\|P_{n-1}\right\|_{\alpha}\left\|f^{(n)}\right\|_{\gamma}\left[(\lambda-m)^{\frac{\beta+1}{\beta}}+(M-\lambda)^{\frac{\beta+1}{\beta}}\right] .}{(\beta+1)^{\frac{1}{\beta}}}
\end{gathered}
$$

I.e. it holds

$$
\begin{aligned}
& \left|\int_{m}^{M} P_{n-1}(t) k(\lambda, t) f^{(n)}(t) d t\right| \\
& \leq \frac{\left\|P_{n-1}\right\|_{\alpha}\left\|f^{(n)}\right\|_{\gamma}}{(\beta+1)^{\frac{1}{\beta}}}\left[(\lambda-m)^{1+\frac{1}{\beta}}+(M-\lambda)^{1+\frac{1}{\beta}}\right], \quad \forall \lambda \in[m, M] .
\end{aligned}
$$

Therefore we get

$$
\begin{aligned}
& \psi_{1} \leq \sup _{x \in H:\|x\|=1} \int_{m-0}^{M}\left|\int_{m}^{M} P_{n-1}(t) k(\lambda, t) f^{(n)}(t) d t\right| d\left\langle E_{\lambda} x, x\right\rangle \\
& \leq\left(\sup _{x \in H:\|x\|=1} \int_{m-0}^{M}\left[(\lambda-m)^{1+\frac{1}{\beta}}+(M-\lambda)^{1+\frac{1}{\beta}}\right] d\left\langle E_{\lambda} x, x\right\rangle\right)
\end{aligned}
$$




$$
\begin{gathered}
\frac{\left\|P_{n-1}\right\|_{\alpha,[m, M]}\left\|f^{(n)}\right\|_{\gamma,[m, M]}}{(\beta+1)^{\frac{1}{\beta}}} \\
\leq\left(\frac{\left\|P_{n-1}\right\|_{\alpha,[m, M]}\left\|f^{(n)}\right\|_{\gamma,[m, M]}}{(\beta+1)^{\frac{1}{\beta}}}\right) \\
{\left[\left\|\left(A-m 1_{H}\right)^{1+\frac{1}{\beta}}\right\|+\left\|\left(M 1_{H}-A\right)^{1+\frac{1}{\beta}}\right\|\right] .}
\end{gathered}
$$

We have proved that

$$
\begin{gathered}
\left\|\int_{m-0}^{M}\left(\int_{m}^{M} P_{n-1}(t) k(\lambda, t) f^{(n)}(t) d t\right) d E_{\lambda}\right\| \\
\leq \frac{\left\|P_{n-1}\right\|_{\alpha,[m, M]}\left\|f^{(n)}\right\|_{\gamma,[m, M]}}{(\beta+1)^{\frac{1}{\beta}}}\left[\left\|\left(A-m 1_{H}\right)^{1+\frac{1}{\beta}}\right\|+\left\|\left(M 1_{H}-A\right)^{1+\frac{1}{\beta}}\right\|\right] .
\end{gathered}
$$

Similarly, it holds

$$
\begin{gathered}
\left\|\int_{m-0}^{M}\left(\int_{m}^{M} P_{n-1}(t) k(\lambda, t) g^{(n)}(t) d t\right) d E_{\lambda}\right\| \\
\leq \frac{\left\|P_{n-1}\right\|_{\alpha,[m, M]}\left\|g^{(n)}\right\|_{\gamma,[m, M]}}{(\beta+1)^{\frac{1}{\beta}}}\left[\left\|\left(A-m 1_{H}\right)^{1+\frac{1}{\beta}}\right\|+\left\|\left(M 1_{H}-A\right)^{1+\frac{1}{\beta}}\right\|\right] .
\end{gathered}
$$

Using (3.23) we derive

$$
\begin{gathered}
|R| \leq \frac{1}{(M-m)}\left\{\|g(A)\| \frac{\left\|P_{n-1}\right\|_{\alpha,[m, M]}\left\|f^{(n)}\right\|_{\gamma,[m, M]}}{(\beta+1)^{\frac{1}{\beta}}}\right. \\
{\left[\left\|\left(A-m 1_{H}\right)^{1+\frac{1}{\beta}}\right\|+\left\|\left(M 1_{H}-A\right)^{1+\frac{1}{\beta}}\right\|\right]} \\
+\|f(A)\| \frac{\left\|P_{n-1}\right\|_{\alpha,[m, M]}\left\|g^{(n)}\right\|_{\gamma,[m, M]}}{(\beta+1)^{\frac{1}{\beta}}} \\
\left.\left[\left\|\left(A-m 1_{H}\right)^{1+\frac{1}{\beta}}\right\|+\left\|\left(M 1_{H}-A\right)^{1+\frac{1}{\beta}}\right\|\right]\right\} \\
{\left[\| \frac{1}{(M-m)}\left[\|g(A)\|\left\|f^{(n)}\right\|\left\|_{\gamma,[m, M]}+\right\| f(A)\left\|g^{(n)}\right\| \|_{\gamma,[m, M]}\right]\right.} \\
\left.\left[A-m 1_{H}\right)^{1+\frac{1}{\beta}}\|+\|\left(M 1_{H}-A\right)^{1+\frac{1}{\beta}} \|\right],
\end{gathered}
$$

proving the claim.

The case $n=1$ follows. 
Corollary 3.4. (to Theorem 3.3) All as in Theorem 3.3. It holds

$$
\begin{gathered}
|\langle f(A) g(A) x, x\rangle-\langle f(A) x, x\rangle\langle g(A) x, x\rangle| \\
\leq \frac{1}{(M-m)(\beta+1)^{\frac{1}{\beta}}}\left[\|g(A)\|\left\|f^{\prime}\right\|_{\gamma,[m, M]}+\|f(A)\|\left\|g^{\prime}\right\|_{\gamma,[m, M]}\right] \\
{\left[\left\|\left(A-m 1_{H}\right)^{1+\frac{1}{\beta}}\right\|+\left\|\left(M 1_{H}-A\right)^{1+\frac{1}{\beta}}\right\|\right] .}
\end{gathered}
$$

We also give

Theorem 3.5. All as in Theorem 3.1. It holds

$$
\begin{gathered}
\langle(\Delta(f, g))(A) x, x\rangle \leq\left\|P_{n-1}\right\|_{\infty,[m, M]} \\
{\left[\|g(A)\|\left\|f^{(n)}\right\|_{1,[m, M]}+\|f(A)\|\left\|g^{(n)}\right\|_{1,[m, M]}\right] .}
\end{gathered}
$$

Proof. We have that

So that

$$
\begin{gathered}
\left|\int_{m}^{M} P_{n-1}(t) k(\lambda, t) f^{(n)}(t) d t\right| \leq \int_{m}^{M}\left|P_{n-1}(t)\right||k(\lambda, t)|\left|f^{(n)}(t)\right| d t \\
\leq\left\|P_{n-1}\right\|_{\infty,[m, M]}(M-m) \int_{m}^{M}\left|f^{(n)}(t)\right| d t \\
=\left\|P_{n-1}\right\|_{\infty,[m, M]}(M-m)\left\|f^{(n)}\right\|_{1,[m, M]}
\end{gathered}
$$

Hence

$$
\begin{gathered}
\left|\int_{m}^{M} P_{n-1}(t) k(\lambda, t) f^{(n)}(t) d t\right| \\
\leq(M-m)\left\|P_{n-1}\right\|_{\infty,[m, M]}\left\|f^{(n)}\right\|_{1,[m, M]} .
\end{gathered}
$$

$$
\begin{gathered}
\left\|\int_{m-0}^{M}\left(\int_{m}^{M} P_{n-1}(t) k(\lambda, t) f^{(n)}(t) d t\right) d E_{\lambda}\right\| \\
=\sup _{x \in H:\|x\|=1}\left|\int_{m-0}^{M}\left(\int_{m}^{M} P_{n-1}(t) k(\lambda, t) f^{(n)}(t) d t\right) d\left\langle E_{\lambda} x, x\right\rangle\right| \\
\leq(M-m)\left\|P_{n-1}\right\|_{\infty,[m, M]}\left\|f^{(n)}\right\|_{1,[m, M]},
\end{gathered}
$$

and similarly,$$
\left\|\int_{m-0}^{M}\left(\int_{m}^{M} P_{n-1}(t) k(\lambda, t) g^{(n)}(t) d t\right) d E_{\lambda}\right\|
$$$$
\leq(M-m)\left\|P_{n-1}\right\|_{\infty,[m, M]}\left\|g^{(n)}\right\|_{1,[m, M]} .
$$

Using (3.23) we obtain

$$
|R| \leq \frac{1}{(M-m)}\left\{\|g(A)\|(M-m)\left\|P_{n-1}\right\|_{\infty,[m, M]}\left\|f^{(n)}\right\|_{1,[m, M]}\right.
$$




$$
\begin{gathered}
\left.+\|f(A)\|(M-m)\left\|P_{n-1}\right\|_{\infty,[m, M]}\left\|g^{(n)}\right\|_{1,[m, M]}\right\} \\
=\left\|P_{n-1}\right\|_{\infty,[m, M]}\left[\|g(A)\|\left\|f^{(n)}\right\|_{1,[m, M]}+\|f(A)\|\left\|g^{(n)}\right\|_{1,[m, M]}\right],
\end{gathered}
$$

proving the claim.

The case $n=1$ follows.

Corollary 3.6. (to Theorem 3.5) It holds

$$
\begin{aligned}
& |\langle f(A) g(A) x, x\rangle-\langle f(A) x, x\rangle\langle g(A) x, x\rangle| \\
\leq & {\left[\|g(A)\|\left\|f^{\prime}\right\|_{1,[m, M]}+\|f(A)\|\left\|g^{\prime}\right\|_{1,[m, M]}\right] . }
\end{aligned}
$$

Comment 3.7. The case of harmonic sequence of polynomials $P_{k}(t)=\frac{(t-x)^{k}}{k !}, k \in \mathbb{Z}_{+}$, was completely studied in [2], and this work generalizes it.

Another harmonic sequence of polynomials related to this work is

$$
P_{k}(t)=\frac{1}{k !}\left(t-\frac{m+M}{2}\right)^{k}, k \in \mathbb{Z}_{+},
$$

see also [4].

The Bernoulli polynomials $B_{n}(t)$ can be defined by the formula (see [4])

$$
\frac{x e^{t x}}{e^{x}-1}=\sum_{n=0}^{\infty} \frac{B_{n}(t)}{n !} x^{n}, \quad|x|<2 \pi, t \in \mathbb{R} .
$$

They satisfy the relation

$$
B_{n}^{\prime}(t)=n B_{n-1}(t), \quad n \in \mathbb{N}
$$

The sequence

$$
P_{n}(t)=\frac{1}{n !} B_{n}(t), \quad n \in \mathbb{Z}_{+},
$$

is a harmonic sequence of polynomials, $t \in \mathbb{R}$.

The Euler polynomials are defined by the formula (see [4])

$$
\frac{2 e^{t x}}{e^{x}+1}=\sum_{n=0}^{\infty} \frac{E_{n}(t)}{n !} x^{n}, \quad|x|<\pi, t \in \mathbb{R} .
$$

They satisfy

$$
E_{n}^{\prime}(t)=n E_{n-1}(t), \quad n \in \mathbb{N}
$$

The sequence

$$
P_{n}(t)=\frac{1}{n !} E_{n}(t), \quad n \in \mathbb{Z}_{+}, t \in \mathbb{R},
$$

is a harmonic sequence of polynomials.

Finally:

Comment 3.8. One can apply (3.3), (3.33) and (3.43), for the harmonic sequences of polynomials defined by (3.49), (3.51) and (3.53). 
In particular, when (see (3.49))

$$
P_{n}(t)=\frac{1}{n !}\left(t-\frac{m+M}{2}\right)^{n}, n \in \mathbb{Z}_{+},
$$

we get

$$
\left\|P_{n-1}\right\|_{\infty,[m, M]}=\frac{1}{(n-1) !}\left(\frac{M-m}{2}\right)^{n-1}
$$

and

$$
\left\|P_{n-1}\right\|_{\alpha,[m, M]}=\frac{1}{(n-1) !(\alpha(n-1)+1)^{\frac{1}{\alpha}}}\left(\frac{(M-m)^{\alpha(n-1)+1}}{2^{\alpha(n-1)}}\right),
$$

where $\alpha, \beta, \gamma>1: \frac{1}{\alpha}+\frac{1}{\beta}+\frac{1}{\gamma}=1$.

\section{References}

[1] Anastassiou, G.A., Intelligent Comparisons: Analytic Inequalities, Springer, New York, Heidelberg, 2016.

[2] Anastassiou, G.A., Self Adjoint Operator Chebyshev-Grüss type inequalities, submitted, 2016.

[3] Čebyšev, P.L., Sur les expressions approximatives des intégrales définies par les autres proses entre les mêmes limites, Proc. Math. Soc. Charkov, 2(1882), 93-98.

[4] Dedić, Lj., Pečarić, J.E., Ujević, N., On generalizations of Ostrowski inequality and some related results, Czechoslovak Mathematical Journal, 53(2003), no. 1, 173-189.

[5] Dragomir, S.S., Inequalities for functions of selfadjoint operators on Hilbert Spaces, ajmaa.org/RGMIA/monographs/InFuncOp.pdf, 2011.

[6] Dragomir, S.S., Operator inequalities of Ostrowski and Trapezoidal type, Springer, New York, 2012.

[7] Fink, A.M., Bounds on the deviation of a function from its averages, Czechoslovak Mathematical Journal, 42(117)(1992), 289-310.

[8] Furuta, T., Mićić Hot, J., Pečaric, J., Seo, Y., Mond-Pečaric Method in Operator Inequalities. Inequalities for Bounded Selfadjoint Operators on a Hilbert Space, Element, Zagreb, 2005.

[9] Grüss, G., Über das Maximum des absoluten Betrages von $\left[\left(\frac{1}{b-a}\right) \int_{a}^{b} f(x) g(x) d x-\left(\frac{1}{(b-a)^{2}} \int_{a}^{b} f(x) d x \int_{a}^{b} g(x) d x\right)\right]$, Math. Z., 39(1935), 215-226.

[10] Helmberg, G., Introduction to Spectral Theory in Hilbert Space, John Wiley \& Sons, Inc., New York, 1969.

George A. Anastassiou

Department of Mathematical Sciences

University of Memphis

Memphis, TN 38152, U.S.A.

e-mail: ganastss@memphis.edu 